\title{
On Idempotent Intuitionistic Fuzzy Matrices of T-Type
}

\author{
Riyaz Ahmad Padder and P. Murugadas \\ Department of Mathematics, Annamalai University, Tamil Nadu, India
}

\section{]jfis}

\begin{abstract}
In this paper, we examine idempotent intuitionistic fuzzy matrices and idempotent intuitionistic fuzzy matrices of T-type. We develop some properties on both idempotent intuitionistic fuzzy matrices and idempotent intuitionistic fuzzy matrices of T-type. Several theorems are provided and an numerical example is given to illustrate the theorems.
\end{abstract}

Keywords: Intuitionistic fuzzy sets, Intuitionistic fuzzy matrix, Idempotent matrix

\section{Introduction}

We are living in a real world where we have to handle situations involving uncertainty, imprecision and vagueness. Moreover the great deal of data involved in Economics, Engineering, Medical science and other fields are not always clear and includes all kinds of uncertainty. But in classical Mathematics all the Mathematical tools for modeling, reasoning and calculations are certain or precise which deal with certain problems. So that they can not solve those complex problems in real situations. In recent years researchers have become interested to deal with the complexity of uncertain data. In 1965 Zadeh [1] came out with the concept of fuzzy set which is indeed an extension of the classical notion of set. However, there are some limitations in dealing with uncertainties by fuzzy sets. To overcome these difficulties, Atanassov [2] developed theory of intuitionistic fuzzy sets as a generalization of fuzzy sets. Jang et al. [3] studied interval-valued intuitionistic fuzzy sets. Park et al. [4] discussed about generalized intuitionistic fuzzy soft set theoretic approach to decision making problem.

A Fuzzy Matrix (FM) is a matrix with elements having values in closed interval $[0,1]$.

Received: Aug. 30, 2016 Revised : Sep. 13, 2016 Accepted: Sep. 19, 2016

Correspondence to: Riyaz Ahmad Padder (padderriyaz01@gmail.com)

(C)The Korean Institute of Intelligent Systems

(c) This is an Open Access article distributed under the terms of the Creative Commons Attribution Non-Commercial License (http://creativecommons.org/licenses/ by-nc/3.0// which permits unrestricted noncommercial use, distribution, and reproduction in any medium, provided the original work is properly cited.
Kim and Roush [5] introduced the concept of FM. FM play a vital role, in various areas in Science and Engineering and solve the problems involving various types of uncertainties [6]. Kim [7] studied inverse of idempotent FMs. Mishref and Emam [8] discussed transitivity and sub-inverse of FMs. Ragab and Emam [9] found determinant and adjoint of a square fuzzy matrices. Kim [10] studied idempotent FM of T-type and developed some properties. Meenakshi [11] studied minus ordering, space ordering and schur complement of FM and block FM. Later much work has been done by many researchers on FM. FMs deal only with membership value where as Intuitionistic Fuzzy Matrices (IFMs) deals with both membership and non membership value. Pal [12] developed the intuitionistic fuzzy determinant. Im et al. [13], studied the determinant of square intuitionistic fuzzy matrices. Pal et al. [14], developed the intuitionistic fuzzy matrices and studied several properties of it using the idea of IFSs and intuitionistic fuzzy determinant. Later, Shyamal and Pal [15] studied some more 
properties on fuzzy matrices. Shyamal and Pal [16] found the distance between IFMs. Bhowmik and Pal [17,-19] developed some results on IFMs, circulant FM and generalized IFMs. Khan and Pal [20] introduced the concept of generalized inverse for IFMs and studied several properties. Adak et al. [21], studied on generalized intuitionistic fuzzy nilpotent matrices over distributive lattice. Several authors [22-27] worked on IFMs and obtained various interesting results, which are very useful in handling uncertainty problems in our daily life. Here we introduce idempotent IFMs of T-type and improve some theorems.

The notion of IFM was given by Atanassov [28]. Further, the concept of IFM was developed by Pal et al. [14] have some special features which are not available in fuzzy matrix theory. Normally fuzzy matrix theory deals only with relevant information, but IFM deals with both relevant and irrelevant information. This motivates us to develop the study on IFM theory, in particular idempotent IFM in analogous to idempotent FM.

\section{Basic Definitions}

Definition 2.1. $[29]$ An Intuitionistic Fuzzy Set (IFS) A in $X$, where $X$ denotes a universal set is defined as an object of the following form $A=\left\{\left\langle x, \mu_{A}(x), \nu_{A}(x)\right\rangle / x \in X\right\}$, where the functions: $\mu_{A}: X \rightarrow[0,1]$ and $\nu_{A}: X \rightarrow[0,1]$ define the membership function and non-membership function of the element $x \in X$ respectively and for every $x \in X: 0 \leq$ $\mu_{A}(x)+\nu_{A}(x) \leq 1$.

Definition 2.2. [14] Let $X=\left\{x_{1}, x_{2}, \ldots x_{m}\right\}$ be a set of alternatives and $Y=\left\{y_{1}, y_{2}, \ldots y_{n}\right\}$ be the attribute set of each element of $X$. An IFM is defined by

$A=\left(\left\langle\left(x_{i}, y_{j}\right), \mu_{A}\left(x_{i}, y_{j}\right), \nu_{A}\left(x_{i}, y_{j}\right)\right\rangle\right)$ for $i=1,2 \ldots m$ and $j=1,2, \ldots n$, where $\mu_{A}: X \times Y \rightarrow[0,1]$ and $\nu_{A}: X \times Y \rightarrow$ $[0,1]$ satisfy the condition $0 \leq \mu_{A}\left(x_{i}, y_{j}\right)+\nu_{A}\left(x_{i}, y_{j}\right) \leq 1$. For simplicity we denote an IFM is a matrix of pairs $A=$ $\left(\left\langle a_{i j}, a_{i j}^{\prime}\right\rangle\right)$ of non negative real numbers satisfying $a_{i j}+a_{i j}^{\prime} \leq$ 1 for all $i, j$. We denote the set of all IFM of order $n \times n$ by $\mathscr{F}_{n}$.

Definition 2.3. [13] The determinant of $|A|$ of $n \times n$ intuitionistic fuzzy matrix $A$ is defined as follows:

$|A|=\left[\left(\bigvee_{\sigma \in S_{n}} a_{1 \sigma(1)} \wedge \ldots \wedge a_{n \sigma(n)}, \bigwedge_{\sigma \in S_{n}} a_{1 \sigma(1)}^{\prime} \vee \ldots \vee a_{n \sigma(n)}^{\prime}\right)\right]$,

where $S_{n}$ denotes the symmetric group of all permutations of the indices $\{1,2, \ldots, n\}$
Definition 2.4. $[7]$ Let $E_{i j} \in \mathscr{F}_{n}$ denotes matrix obtained from the identity matrix $I=I_{n}$ by interchanging row $i$ and row $j(i \leq j)$

Some of the definitions and results we apply in this paper are give below.

Let $\mathrm{A}$ and $\mathrm{B}$ be an $n \times n$ IFMs with elements from closed interval $[0,1] \times[0,1]$ such that $A=\left(\left\langle a_{i j}, a_{i j}^{\prime}\right\rangle\right)$ and $B=$ $\left(\left\langle b_{i j}, b_{i j}^{\prime}\right\rangle\right)$ respectively.

$A \vee B=\left(\left\langle a_{i j}, a_{i j}^{\prime}\right\rangle\right) \vee\left(\left\langle b_{i j}, b_{i j}^{\prime}\right\rangle\right)=\left(\left\langle a_{i j} \vee b_{i j}, a_{i j}^{\prime} \wedge b_{i j}^{\prime}\right\rangle\right)$,

$A \wedge B=\left(\left\langle a_{i j}, a_{i j}^{\prime}\right\rangle\right) \wedge\left(\left\langle b_{i j}, b_{i j}^{\prime}\right\rangle\right)=\left(\left\langle a_{i j} \wedge b_{i j}, a_{i j}^{\prime} \wedge b_{i j}^{\prime}\right\rangle\right)$,

$A \times B=\left[\left(\left\langle a_{i 1} \wedge b_{1 j}, a_{i 1}^{\prime} \vee b_{1 j}^{\prime}\right\rangle\right) \vee\left(\left\langle a_{i 2} \wedge b_{2 j}, a_{i 2}^{\prime} \vee b_{2 j}^{\prime}\right\rangle\right) \vee\right.$

$\left.\ldots \vee\left(\left\langle a_{i n} \wedge b_{n j}, a_{i n}^{\prime} \vee b_{n j}^{\prime}\right\rangle\right)\right]$,

$A^{k+1}=A^{k} \times A, \quad(\mathrm{k}=0,1,2, \ldots)$,

$A^{0}=I=\left[\delta_{i j}, \delta_{i j}^{\prime}\right]$, (where $\left[\delta_{i j}, \delta_{i j}^{\prime}\right]$ is Kroneker delta),

$A^{T}=\left[q_{j i}, q_{j i}^{\prime}\right]$ (Transpose of A)

$A \leq B$ iff $\left(\left\langle a_{i j}, a_{i j}^{\prime}\right\rangle \leq\left\langle b_{i j}, b_{i j}^{\prime}\right\rangle\right.$ for all $\left.i, j \in\{1,2, \ldots, n\}\right)$. The IFM A is said to be,

(a) Symmetric if $A=A^{T}$.

(b) Idempotent if $A^{2}=A$

(c) (Max-min) transitive if $A^{2} \leq A$

(d) Nilpotent if $A^{n}=(\langle 0,1\rangle)$

\section{Results}

Definition 3.1. Let $A=\left(\left\langle a_{i j}, a_{i j}^{\prime}\right\rangle\right) \in \mathscr{F}_{n}$. A is said to be normal if $\left\langle a_{11}, a_{11}^{\prime}\right\rangle \geq\left\langle a_{22}, a_{22}^{\prime}\right\rangle \geq \ldots \geq\left\langle a_{n n}, a_{n n}^{\prime}\right\rangle$.

Theorem 3.2. Let $A=\left(\left\langle a_{i j}, a_{i j}^{\prime}\right\rangle\right)$ be an idempotent IFM. If A is normal, then

(1) $\left\langle a_{i j}, a_{i j}^{\prime}\right\rangle \leq\left\langle a_{11}, a_{11}^{\prime}\right\rangle$ for all $i, j \in\{1,2, \ldots, n\}$

(2) Let $\min \left\{\left\langle a_{i j}, a_{i j}^{\prime}\right\rangle: i, j \in\{1,2, \ldots, n\}\right\}=\left\langle c, c^{\prime}\right\rangle$.

If $\left\langle c, c^{\prime}\right\rangle=\left\langle a_{i j}, a_{i j}^{\prime}\right\rangle(i \neq j)$, then there exists $\left\langle a_{i n}, a_{i n}^{\prime}\right\rangle$ or $\left\langle a_{n j}, a_{n j}^{\prime}\right\rangle$ such that $\left\langle c, c^{\prime}\right\rangle=\left\langle a_{i n}, a_{i n}^{\prime}\right\rangle$ or $\left\langle c, c^{\prime}\right\rangle=\left\langle a_{n j}, a_{n j}^{\prime}\right\rangle$.

Proof. (1) In order to prove $\left\langle a_{i j}, a_{i j}^{\prime}\right\rangle \leq\left\langle a_{11}, a_{11}^{\prime}\right\rangle$ we have to first show that $\left\langle a_{1 n}, a_{1 n}^{\prime}\right\rangle \leq\left\langle a_{11}, a_{11}^{\prime}\right\rangle$.

Suppose that $\left\langle a_{1 n}, a_{1 n}^{\prime}\right\rangle>\left\langle a_{11}, a_{11}^{\prime}\right\rangle$ (we shall obtain contradiction. Put $\left\langle a_{1 n}, a_{1 n}^{\prime}\right\rangle=\left\langle r, r^{\prime}\right\rangle$.

The proof consists of following steps.

(a) $\mathrm{A}$ is idempotent $\Rightarrow A A=A$, we have that

$\left\langle a_{1 n}, a_{1 n}^{\prime}\right\rangle=\sum_{t=1}^{n}\left\langle a_{1 t}, a_{1 t}^{\prime}\right\rangle\left\langle a_{t n}, a_{t n}^{\prime}\right\rangle$ and

$\left\langle a_{1 k}, a_{1 k}^{\prime}\right\rangle\left\langle a_{k n}, a_{k n}^{\prime}\right\rangle=\left\langle a_{1 n}, a_{1 n}^{\prime}\right\rangle$ for $1<k<n$.

If $\mathrm{k}=1$, then $\left\langle a_{11}, a_{11}^{\prime}\right\rangle\left\langle a_{1 n}, a_{1 n}^{\prime}\right\rangle=\left\langle a_{1 n}, a_{1 n}^{\prime}\right\rangle \leq\left\langle a_{11}, a_{11}^{\prime}\right\rangle$, contrary to $\left\langle a_{1 n}, a_{1 n}^{\prime}\right\rangle>\left\langle a_{11}, a_{11}^{\prime}\right\rangle$.

Similarly we can prove that $k \neq n$.

If $\left\langle a_{n k}, a_{n k}^{\prime}\right\rangle \geq\left\langle r, r^{\prime}\right\rangle \Rightarrow\left\langle a_{n k}, a_{n k}^{\prime}\right\rangle\left\langle a_{k n}, a_{k n}^{\prime}\right\rangle \geq\left\langle r, r^{\prime}\right\rangle$, contrary to $\left\langle a_{n k}, a_{n k}^{\prime}\right\rangle\left\langle a_{k n}, a_{k n}^{\prime}\right\rangle \leq\left\langle a_{n n}, a_{n n}^{\prime}\right\rangle \leq\left\langle a_{11}, a_{11}^{\prime}\right\rangle<$ 
$\left\langle r, r^{\prime}\right\rangle$ we get,

$\left\langle a_{1 n}, a_{1 n}^{\prime}\right\rangle=\left\langle a_{1 k}, a_{1 k}^{\prime}\right\rangle\left\langle a_{k n}, a_{k n}^{\prime}\right\rangle, 1<k<n$ and $\left\langle a_{n k}, a_{n k}^{\prime}\right\rangle<\left\langle r, r^{\prime}\right\rangle$.

(b) Suppose that $\left\langle a_{1 k}, a_{1 k}^{\prime}\right\rangle$ is the first such element from $\left\langle a_{11}, a_{11}^{\prime}\right\rangle$ with the property $\left\langle a_{1 n}, a_{1 n}^{\prime}\right\rangle=\left\langle a_{1 k}, a_{1 k}^{\prime}\right\rangle\left\langle a_{k n}, a_{k n}^{\prime}\right\rangle$ (and there is no $t$ such that $1<t<k$ and $\left\langle a_{1 n}, a_{1 n}^{\prime}\right\rangle=$ $\left.\left\langle a_{1 t}, a_{1 t}^{\prime}\right\rangle\left\langle a_{t n}, a_{t n}^{\prime}\right\rangle\right)$

Note that $\left\langle a_{1 k}, a_{1 k}^{\prime}\right\rangle \geq\left\langle r, r^{\prime}\right\rangle$.

Since $A A=A$, for $\left\langle a_{1 k}, a_{1 k}^{\prime}\right\rangle$ there exists $\left\langle a_{1 u}, a_{1 u}^{\prime}\right\rangle$ such that $\left\langle a_{1 k}, a_{1 k}^{\prime}\right\rangle=\left\langle a_{1 u}, a_{1 u}^{\prime}\right\rangle\left\langle a_{u k}, a_{u k}^{\prime}\right\rangle$ and $k \leq u \leq n$.

We suppose that $\left\langle a_{1 u}, a_{1 u}^{\prime}\right\rangle$ is the first one from $\left\langle a_{1 k}, a_{1 k}^{\prime}\right\rangle$ with this property.

If $u=k$, then $\left\langle a_{k k}, a_{k k}^{\prime}\right\rangle \geq\left\langle r, r^{\prime}\right\rangle$, which contradicts $\left\langle a_{k k}, a_{k k}^{\prime}\right\rangle \leq\left\langle a_{11}, a_{11}^{\prime}\right\rangle\left\langle\left\langle a_{1 n}, a_{1 n}^{\prime}\right\rangle=\left\langle r, r^{\prime}\right\rangle\right.$.

Thus, we have $k<u$. If $u=n, \Rightarrow\left\langle a_{n k}, a_{n k}^{\prime}\right\rangle\left\langle a_{1 k}, a_{1 k}^{\prime}\right\rangle \geq$ $\left\langle r, r^{\prime}\right\rangle$.

But we know from $\left\langle a_{1 n}, a_{1 n}^{\prime}\right\rangle=\left\langle a_{1 k}, a_{1 k}^{\prime}\right\rangle\left\langle a_{k n}, a_{k n}^{\prime}\right\rangle$ that $\left\langle a_{k n}, a_{k n}^{\prime}\right\rangle \geq\left\langle r, r^{\prime}\right\rangle$.

Then we obtain that $\left\langle a_{n k}, a_{n k}^{\prime}\right\rangle\left\langle a_{k n}, a_{k n}^{\prime}\right\rangle \geq\left\langle r, r^{\prime}\right\rangle$, contradicts with $\left\langle a_{n n}, a_{n n}^{\prime}\right\rangle \leq\left\langle a_{11}, a_{11}^{\prime}\right\rangle<\left\langle r, r^{\prime}\right\rangle$. We say that $\left\langle a_{1 k}, a_{1 k}^{\prime}\right\rangle=\left\langle a_{1 u}, a_{1 u}^{\prime}\right\rangle\left\langle a_{u k}, a_{u k}^{\prime}\right\rangle$ with $k<u<n$.

(c) Using the above argument for $\left\langle a_{1 u}, a_{1 u}^{\prime}\right\rangle$,

we get that $\left\langle a_{1 u}, a_{1 u}^{\prime}\right\rangle=\left\langle a_{1 v}, a_{1 v}^{\prime}\right\rangle\left\langle a_{v u}, a_{v u}^{\prime}\right\rangle$ with $1<k<$ $u<v<n$.

(If $v=u$, then

$\left\langle a_{1 u}, a_{1 u}^{\prime}\right\rangle=\left\langle a_{1 u}, a_{1 u}^{\prime}\right\rangle\left\langle a_{u u}, a_{u u}^{\prime}\right\rangle \leq\left\langle a_{u u}, a_{u u}^{\prime}\right\rangle \leq$

$\left\langle a_{11}, a_{11}^{\prime}\right\rangle<\left\langle r, r^{\prime}\right\rangle$, contrary to

$\left\langle r, r^{\prime}\right\rangle=\left\langle a_{1 n}, a_{1 n}^{\prime}\right\rangle=\left\langle a_{1 k}, a_{1 k}^{\prime}\right\rangle\left\langle a_{k n}, a_{k n}^{\prime}\right\rangle=$

$\left\langle a_{1 u}, a_{1 u}^{\prime}\right\rangle\left\langle a_{u k}, a_{u k}^{\prime}\right\rangle\left\langle a_{k n}, a_{k n}^{\prime}\right\rangle$ and $\left.\left\langle a_{1 u}, a_{1 u}^{\prime}\right\rangle \geq\left\langle r, r^{\prime}\right\rangle\right)$.

(If $v=n$, then

$\left\langle a_{1 n}, a_{1 n}^{\prime}\right\rangle=\left\langle a_{1 k}, a_{1 k j}^{\prime}\right\rangle\left\langle a_{k n}, a_{k n}^{\prime}\right\rangle=$

$\left\langle a_{1 u}, a_{1 u}^{\prime}\right\rangle\left\langle a_{u k}, a_{u k}^{\prime}\right\rangle\left\langle a_{k n}, a_{k n}^{\prime}\right\rangle=$

$\left\langle a_{1 n}, a_{1 n}^{\prime}\right\rangle\left\langle a_{n u}, a_{n u}^{\prime}\right\rangle\left\langle a_{u k}, a_{u k}^{\prime}\right\rangle\left\langle a_{k n}, a_{k n}^{\prime}\right\rangle$ and

$\left\langle a_{n u}, a_{n u}^{\prime}\right\rangle\left\langle a_{u k}, a_{u k}^{\prime}\right\rangle\left\langle a_{k n}, a_{k n}^{\prime}\right\rangle \geq\left\langle r, r^{\prime}\right\rangle$, contrary to

$\left\langle a_{n u}, a_{n u}^{\prime}\right\rangle\left\langle a_{u k}, a_{u k}^{\prime}\right\rangle\left\langle a_{k n}, a_{k n}^{\prime}\right\rangle \leq\left\langle a_{n n}, a_{n n}^{\prime}\right\rangle<\left\langle r, r^{\prime}\right\rangle$. Thus, $v=n)$.

(d) We put $k=k(1), u=k(2)$ and $v=k(3)$. We get, $1<k(1)<k(2)<k(3)<n$,

$\left\langle a_{1 k(1)}, a_{1 k(1)}^{\prime}\right\rangle=$

$\left\langle a_{1 k(2)}, a_{1 k(2)}^{\prime}\right\rangle\left\langle a_{k(1) k(2)}, a_{k(1) k(2)}^{\prime}\right\rangle,\left\langle a_{1 k(2)}, a_{1 k(2)}^{\prime}\right\rangle$

$=\left\langle a_{1 k(3)}, a_{1 k(3)}^{\prime}\right\rangle\left\langle a_{k(3) k(2)}, a_{k(3) k(2)}^{\prime}\right\rangle$,

$\left\langle a_{1 n}, a_{1 n}^{\prime}\right\rangle=\left\langle a_{1 k(1)}, a_{1 k(1)}^{\prime}\right\rangle\left\langle a_{k(1) n}, a_{k(1) n}^{\prime}\right\rangle$, and

$K=\left\{\left\langle a_{1 k(t)}, a_{1 k(t)}^{\prime}\right\rangle: t=1,2,3\right\}$.

We note that $\left\langle a_{1 k(t)}, a_{1 k(t)}^{\prime}\right\rangle \geq\left\langle r, r^{\prime}\right\rangle$ for $\left\langle a_{1 k(t)}, a_{1 k(t)}^{\prime}\right\rangle \in K$

(e) If we carry on the argument (a), (b) and (c) then the number of the elements $\left\langle a_{1 k(t)}, a_{1 k(t)}^{\prime}\right\rangle$ of the set $K$ increases indefi- nitely, but the cardinal number of $\left\{\left\langle a_{1 i}, a_{1 i}^{\prime}\right\rangle, i=1,2, \ldots, n\right\}$ is equal to $\mathrm{n}$.

This proves that $\left\langle a_{1 n}, a_{1 n}^{\prime}\right\rangle \leq\left\langle a_{11}, a_{11}^{\prime}\right\rangle$.

(f) At this stage, we just show how to continue with the argument in (a), (b) and (c).

We shall show that

$\left\langle a_{1(n-1)}, a_{1(n-1)}^{\prime}\right\rangle \leq\left\langle a_{11}, a_{11}^{\prime}\right\rangle,\left\langle a_{1(n-2)}, a_{1(n-2)}^{\prime}\right\rangle \leq$ $\left\langle a_{11}, a_{11}^{\prime}\right\rangle, \ldots,\left\langle a_{13}, a_{13}^{\prime}\right\rangle \leq\left\langle a_{11}, a_{11}^{\prime}\right\rangle$ and

$\left\langle a_{12}, a_{12}^{\prime}\right\rangle \leq\left\langle a_{11}, a_{11}^{\prime}\right\rangle$.

Then we take $A^{T}$ and using the above statement, we have $\left\langle a_{t 1}, a_{t 1}^{\prime}\right\rangle \leq\left\langle a_{11}, a_{11}^{\prime}\right\rangle$ for $t=n-1, n-2, \ldots, 2$.

Then we take the second row of $\mathrm{A}$ and prove that

$\left\langle a_{2 n}, a_{2 n}^{\prime}\right\rangle \leq\left\langle a_{11}, a_{11}^{\prime}\right\rangle$.

Then we show that

$\left\langle a_{2(n-1)}, a_{2(n-1)}^{\prime}\right\rangle \leq\left\langle a_{11}, a_{11}^{\prime}\right\rangle, \ldots,\left\langle a_{2(n-1)}, a_{2(n-1)}^{\prime}\right\rangle \leq$ $\left\langle a_{11}, a_{11}^{\prime}\right\rangle$, and so on. This proves (i).

(2) Let $\left\langle c, c^{\prime}\right\rangle=\left\langle a_{i j}, a_{i j}^{\prime}\right\rangle$ with $i \neq n$ and $i \neq n$.

Then we see that

$\left\langle c, c^{\prime}\right\rangle=\left\langle a_{i j}, a_{i j}^{\prime}\right\rangle=\sum_{t=1}^{n}\left\langle a_{i t}, a_{i t}^{\prime}\right\rangle\left\langle a_{t i}, a_{t i}^{\prime}\right\rangle=$

$\left\langle a_{i j}, a_{i j}^{\prime}\right\rangle\left\langle a_{i j}, a_{i j}^{\prime}\right\rangle+\left\langle a_{i j}, a_{i j}^{\prime}\right\rangle\left\langle a_{i j}, a_{i j}^{\prime}\right\rangle+\ldots+\left\langle a_{i j}, a_{i j}^{\prime}\right\rangle\left\langle a_{i j}, a_{i j}^{\prime}\right\rangle$ and $\left\langle a_{i j}, a_{i j}^{\prime}\right\rangle\left\langle a_{i j}, a_{i j}^{\prime}\right\rangle \leq\left\langle c, c^{\prime}\right\rangle$.

We also see that

$\left\langle a_{i j}, a_{i j}^{\prime}\right\rangle \geq\left\langle c, c^{\prime}\right\rangle,\left\langle a_{i j}, a_{i j}^{\prime}\right\rangle \geq\left\langle c, c^{\prime}\right\rangle$, and

$\left\langle a_{i j}, a_{i j}^{\prime}\right\rangle\left\langle a_{i j}, a_{i j}^{\prime}\right\rangle \geq\left\langle c, c^{\prime}\right\rangle$.

Thus, we get

$\left\langle a_{i j}, a_{i j}^{\prime}\right\rangle\left\langle a_{i j}, a_{i j}^{\prime}\right\rangle=\left\langle c, c^{\prime}\right\rangle$, which deduces that

$\left\langle a_{i j}, a_{i j}^{\prime}\right\rangle=\left\langle c, c^{\prime}\right\rangle$ or $\left\langle a_{i j}, a_{i j}^{\prime}\right\rangle=\left\langle c, c^{\prime}\right\rangle$.

Definition 3.3. (a) Let $A \in \mathscr{F}_{n}$. $|A|$ denote the determinant of $A$.

(b) $[30] \operatorname{adj}(A)=B=\left(\left\langle b_{i j}, b_{i j}^{\prime}\right\rangle\right)$, denote the adjoint matrix of $A$, defined as follows: $\left\langle b_{i j}, b_{i j}^{\prime}\right\rangle=\left|A_{j i}\right|$ where $A_{j i}$ is the $(n-1) \times(n-1)$ IFM formed by deleting row $j$ and column $i$ from $A$.

(c) Let $A=\left(\left\langle a_{i j}, a_{i j}^{\prime}\right\rangle\right)$ be an idempotent IFM, and assume that $A$ is normal. $A$ is said to be T-type if $\left\langle a_{i t}, a_{i t}^{\prime}\right\rangle \leq\left\langle a_{i i}, a_{i i}^{\prime}\right\rangle$ and $\left\langle a_{t i}, a_{t i}^{\prime}\right\rangle \leq\left\langle a_{i i}, a_{i i}^{\prime}\right\rangle$ for $t \geq i$

Theorem 3.4. Let $A=\left(\left\langle a_{i j}, a_{i j}^{\prime}\right\rangle\right) \in \mathscr{F}_{n}$ be an intuitionistic fuzzy idempotent matrix of T-type, and assume that $A$ is normal. Then $\operatorname{adj}(A)$ is an idempotent IFM.

Proof. The proof consists of following steps

(a) By computations, it is not difficult to find that $\operatorname{adj}(A)=$ $B=\left(\left\langle b_{i j}, b_{i j}^{\prime}\right\rangle\right)$ takes the form

$\left\langle b_{n n}, b_{n n}^{\prime}\right\rangle=\left\langle a_{n-1}, a_{n-1}^{\prime}\right\rangle$

$\left\langle b_{t t}, b_{t t}^{\prime}\right\rangle=\left\langle a_{n n}, a_{n n}^{\prime}\right\rangle$ if $t \neq n$, 


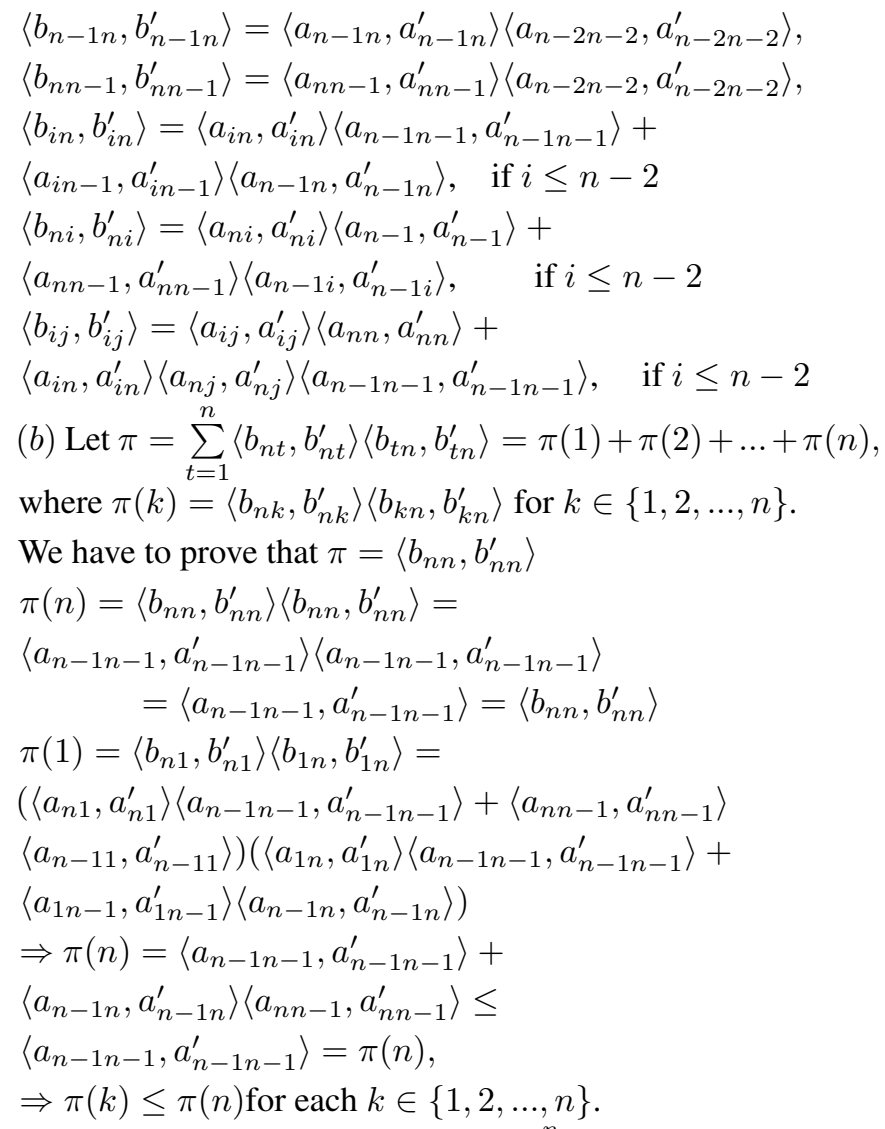

(b) Let $\pi=\sum_{t=1}^{n}\left\langle b_{n t}, b_{n t}^{\prime}\right\rangle\left\langle b_{t n}, b_{t n}^{\prime}\right\rangle=\pi(1)+\pi(2)+\ldots+\pi(n)$, where $\pi(k)=\left\langle b_{n k}, b_{n k}^{\prime}\right\rangle\left\langle b_{k n}, b_{k n}^{\prime}\right\rangle$ for $k \in\{1,2, \ldots, n\}$.

We have to prove that $\pi=\left\langle b_{n n}, b_{n n}^{\prime}\right\rangle$

$$
\begin{aligned}
& \pi(n)=\left\langle b_{n n}, b_{n n}^{\prime}\right\rangle\left\langle b_{n n}, b_{n n}^{\prime}\right\rangle= \\
& \left\langle a_{n-1 n-1}, a_{n-1 n-1}^{\prime}\right\rangle\left\langle a_{n-1 n-1}, a_{n-1 n-1}^{\prime}\right\rangle \\
& \quad=\left\langle a_{n-1 n-1}, a_{n-1 n-1}^{\prime}\right\rangle=\left\langle b_{n n}, b_{n n}^{\prime}\right\rangle \\
& \pi(1)=\left\langle b_{n 1}, b_{n 1}^{\prime}\right\rangle\left\langle b_{1 n}, b_{1 n}^{\prime}\right\rangle= \\
& \left(\left\langle a_{n 1}, a_{n 1}^{\prime}\right\rangle\left\langle a_{n-1 n-1}, a_{n-1 n-1}^{\prime}\right\rangle+\left\langle a_{n n-1}, a_{n n-1}^{\prime}\right\rangle\right. \\
& \left.\left\langle a_{n-11}, a_{n-11}^{\prime}\right\rangle\right)\left(\left\langle a_{1 n}, a_{1 n}^{\prime}\right\rangle\left\langle a_{n-1 n-1}, a_{n-1 n-1}^{\prime}\right\rangle+\right. \\
& \left.\left\langle a_{1 n-1}, a_{1 n-1}^{\prime}\right\rangle\left\langle a_{n-1 n}, a_{n-1 n}^{\prime}\right\rangle\right) \\
& \Rightarrow \pi(n)=\left\langle a_{n-1 n-1}, a_{n-1 n-1}^{\prime}\right\rangle+ \\
& \left\langle a_{n-1 n}, a_{n-1 n}^{\prime}\right\rangle\left\langle a_{n n-1}, a_{n n-1}^{\prime}\right\rangle \leq \\
& \left\langle a_{n-1 n-1}, a_{n-1 n-1}^{\prime}\right\rangle=\pi(n), \\
& \Rightarrow \pi(k) \leq \pi(n) \text { for each } k \in\{1,2, \ldots, n\} .
\end{aligned}
$$

Therefore, we get $\pi=\pi(n)$ and $\sum_{t=1}^{n}\left\langle b_{n t}, b_{n t}^{\prime}\right\rangle\left\langle b_{t n}, b_{t n}^{\prime}\right\rangle=$ $\left\langle b_{n n}, b_{n n}^{\prime}\right\rangle$.

(c) Let $\pi=\sum_{t=1}^{n}\left\langle b_{k t}, b_{k t}^{\prime}\right\rangle\left\langle b_{t k}, b_{t k}^{\prime}\right\rangle=\pi(1)+\pi(2)+\ldots+\pi(n)$, for $1 \leq k \leq n-2$,

where $\pi(i)=\left\langle b_{k i}, b_{k i}^{\prime}\right\rangle\left\langle b_{i k}, b_{i k}^{\prime}\right\rangle$.

We have to prove that $\pi=\left\langle b_{k k}, b_{k k}^{\prime}\right\rangle$.

In order to prove this we take $\pi(k)=\left\langle b_{k k}, b_{k k}^{\prime}\right\rangle\left\langle b_{k k}, b_{k k}^{\prime}\right\rangle$.

We have $\left\langle b_{k k}, b_{k k}^{\prime}\right\rangle\left\langle b_{k k}, b_{k k}^{\prime}\right\rangle=\left\langle a_{n n}, a_{n n}^{\prime}\right\rangle\left\langle a_{n n}, a_{n n}^{\prime}\right\rangle=$ $\left\langle a_{n n}, a_{n n}^{\prime}\right\rangle=\left\langle b_{k k}, b_{k k}^{\prime}\right\rangle$ for $1 \leq k \leq n-2$.

We also can see that $\pi(i) \leq\left\langle a_{n n}, a_{n n}^{\prime}\right\rangle$ for each $i \neq k$, therefore $\pi=\left\langle b_{k k}, b_{k k}^{\prime}\right\rangle$.

It is easy to show that if $k=n-1$, then

$\sum_{t=1}^{n}\left\langle b_{k t}, b_{k t}^{\prime}\right\rangle\left\langle b_{t k}, b_{t k}^{\prime}\right\rangle=\left\langle a_{n n}, a_{n n}^{\prime}\right\rangle=\left\langle b_{k k}, b_{k k}^{\prime}\right\rangle$ and

$\sum_{t=1}^{n}\left\langle b_{n-1 t}, b_{n-1 t}^{\prime}\right\rangle\left\langle b_{t n-1}, b_{t n-1}^{\prime}\right\rangle=\left\langle b_{n-1 n-1}, b_{n-1 n-1}^{\prime}\right\rangle$

(d) Let $\pi=\sum_{t=1}^{n}\left\langle b_{i t}, b_{i t}^{\prime}\right\rangle\left\langle b_{t j}, b_{t j}^{\prime}\right\rangle=\pi(1)+\pi(2)+\ldots+\pi(n)$, for $i, j \leq n-1$,

where $\pi(k)=\left\langle b_{i k}, b_{i k}^{\prime}\right\rangle\left\langle b_{k j}, b_{k j}^{\prime}\right\rangle$, we shall show that $\pi=$ $\left\langle b_{i j}, b_{i j}^{\prime}\right\rangle$.

Let $i<j$. We first suppose that $\mathrm{i}, j \leq n-2$.

We can see that

$\pi(n)=\left\langle b_{i n}, b_{i n}^{\prime}\right\rangle\left\langle b_{n j}, b_{n j}^{\prime}\right\rangle=$ $\left(\left\langle a_{i n}, b_{i n}^{\prime}\right\rangle\left\langle a_{n-1 n-1}, a_{n-1 n-1}^{\prime}\right\rangle+\right.$

$\left.\left\langle a_{i n-1}, a_{i n-1}^{\prime}\right\rangle\left\langle a_{n-1 n}, a_{n-1 n}^{\prime}\right\rangle\right)\left(\left\langle a_{n j}, a_{n j}^{\prime}\right\rangle\right.$

$\left.\left\langle a_{n-1 n-1}, a_{n-1 n-1}^{\prime}\right\rangle+\left\langle a_{n n-1}, a_{n n-1}^{\prime}\right\rangle\left\langle a_{n-1 j}, a_{n-1 j}^{\prime}\right\rangle\right)=$

$\left\langle a_{i n}, a_{i n}^{\prime}\right\rangle\left\langle a_{n j}, a_{n j}^{\prime}\right\rangle\left\langle a_{n-1 n-1}, a_{n-1 n-1}^{\prime}\right\rangle$,

$\pi(i)=\left\langle b_{i i}, b_{i i}^{\prime}\right\rangle\left\langle b_{i j}, b_{i j}^{\prime}\right\rangle=\left\langle a_{n n}, a_{n n}^{\prime}\right\rangle\left(\left\langle a_{i j}, a_{i j}^{\prime}\right\rangle\left\langle a_{n n}, a_{n n}^{\prime}\right\rangle+\right.$

$\left.\left\langle a_{n-1 n-1}, a_{n-1 n-1}^{\prime}\right\rangle\left\langle a_{i n}, a_{i n}^{\prime}\right\rangle\left\langle a_{n j}, a_{n j}^{\prime}\right\rangle\right)=$

$\left\langle a_{i j}, a_{i j}^{\prime}\right\rangle\left\langle a_{n n}, a_{n n}^{\prime}\right\rangle+\left\langle a_{n n}, a_{n n}^{\prime}\right\rangle\left\langle a_{i n}, a_{i n}^{\prime}\right\rangle\left\langle a_{n j}, a_{n j}^{\prime}\right\rangle$, and

$\pi(i)+\pi(n)=$

$\left\langle a_{n n}, a_{n n}^{\prime}\right\rangle\left\langle a_{i j}, a_{i j}^{\prime}\right\rangle+\left\langle a_{n-1 n-1}, a_{k k}^{\prime}\right\rangle\left\langle a_{i n}, a_{i n}^{\prime}\right\rangle\left\langle a_{n j}, a_{n j}^{\prime}\right\rangle=$ $\left\langle b_{i j}, b_{i j}^{\prime}\right\rangle$.

We can prove that

$\pi(k) \leq\left\langle a_{n n}, a_{n n}^{\prime}\right\rangle\left\langle a_{i j}, a_{i j}^{\prime}\right\rangle+$

$\left\langle a_{n-1 n-1}, a_{n-1 n-1}^{\prime}\right\rangle\left\langle a_{i n}, a_{i n}^{\prime}\right\rangle\left\langle a_{n j}, a_{n j}^{\prime}\right\rangle=\left\langle b_{i j}, b_{i j}^{\prime}\right\rangle$ for each $k \in\{1,2, \ldots, n\}$.

Therefore, we have proved that $\sum_{t=1}^{n}\left\langle b_{i t}, b_{i t}^{\prime}\right\rangle\left\langle b_{t j}, b_{t j}^{\prime}\right\rangle=\left\langle b_{i j}, b_{i j}^{\prime}\right\rangle$ for $i, j \leq n-2$.

Similarly, we can prove that $\sum_{t=1}^{n}\left\langle b_{i t}, b_{i t}^{\prime}\right\rangle\left\langle b_{t j}, b_{t j}^{\prime}\right\rangle=\left\langle b_{i j}, b_{i j}^{\prime}\right\rangle$ for $j<n-1$.

(e) Let $\pi=\sum_{t=1}^{n}\left\langle b_{n-1 t}, b_{n-1 t}^{\prime}\right\rangle\left\langle b_{t n}, b_{t n}^{\prime}\right\rangle=\pi(1)+\pi(2)+\ldots+$ $\pi(n)$,

where $\pi(k)=\left\langle b_{n-1 k}, b_{n-1 k}^{\prime}\right\rangle\left\langle b_{k n}, b_{k n}^{\prime}\right\rangle$

Then we shall show that $\pi=\left\langle b_{n-1 n}, b_{n-1 n}^{\prime}\right\rangle$.

We take $\pi(n)=\left\langle b_{n-1 n}, b_{n-1 n}^{\prime}\right\rangle\left\langle b_{n n}, b_{n n}^{\prime}\right\rangle$, and we can see that $\pi(n)=\left(\left\langle a_{n-1 n-1}, a_{n-1 n-1}^{\prime}\right\rangle\left\langle a_{n-1 n}, a_{n-1 n}^{\prime}\right\rangle\right)\left\langle b_{n n}, b_{n n}^{\prime}\right\rangle=$ $\left\langle b_{n-1 n}, b_{n-1 n}^{\prime}\right\rangle$.

$\Rightarrow \pi(n-1)=\left\langle b_{n-1 n-1}, b_{n-1 n-1}^{\prime}\right\rangle\left\langle b_{n-1 n}, b_{n-1 n}^{\prime}\right\rangle=$ $\left\langle a_{n n}, a_{n n}^{\prime}\right\rangle\left\langle a_{n-1 n-1}, a_{n-1 n-1}^{\prime}\right\rangle\left\langle a_{n-1 n}, a_{n-1 n}^{\prime}\right\rangle \leq \pi(n)$, $\pi(1)=\left\langle b_{n-11}, b_{n-11}^{\prime}\right\rangle\left\langle b_{1 n}, b_{1 n}^{\prime}\right\rangle=$

$\left(\left\langle a_{n n}, a_{n n}^{\prime}\right\rangle\left\langle a_{n-11}, a_{n-11}^{\prime}\right\rangle+\left\langle a_{n-1 n-1}, a_{n-1 n-1}^{\prime}\right\rangle\right.$

$\left.\left\langle a_{n-1 n}, a_{n-1 n}^{\prime}\right\rangle\left\langle a_{n 1}, a_{n 1}^{\prime}\right\rangle\right)\left(\left\langle a_{n n}, a_{n n}^{\prime}\right\rangle\left\langle a_{1 n}, a_{1 n}^{\prime}\right\rangle+\right.$

$\left.\left\langle a_{n-1 n-1}, a_{n-1 n-1}^{\prime}\right\rangle\left\langle a_{1 n}, a_{1 n}^{\prime}\right\rangle\left\langle a_{n n}, a_{n n}^{\prime}\right\rangle\right)=\pi(n-1)$, and $\pi(i) \leq \pi(n)$ for $1 \leq i \leq n-1$.

Thus, we have proved that $\pi=\left\langle b_{n-1 n}, b_{n-1 n}^{\prime}\right\rangle$.

Similarly, we can show that $\pi=\left\langle b_{n n-1}, b_{n n-1}^{\prime}\right\rangle$.

$(f)$ Let $\pi=\sum_{t=1}^{n}\left\langle b_{k t}, b_{k t}^{\prime}\right\rangle\left\langle b_{t n}, b_{t n}^{\prime}\right\rangle=\pi(1)+\pi(2)+\ldots+\pi(n)$,

for $k \leq n-1$, where $\pi=\left\langle b_{k t}, b_{k t}^{\prime}\right\rangle\left\langle b_{t n}, b_{t n}^{\prime}\right\rangle$.

$\Rightarrow \pi(n)=\left\langle b_{k n}, b_{k n}^{\prime}\right\rangle\left\langle b_{n n}, b_{n n}^{\prime}\right\rangle=$

$\left\langle b_{k n}, b_{k n}^{\prime}\right\rangle\left\langle a_{n-1 n-1}, a_{n-1 n-1}^{\prime}\right\rangle=$

$\left(\left\langle a_{n-1 n-1}, a_{n-1 n-1}^{\prime}\right\rangle\left\langle a_{k n}, a_{k n}^{\prime}\right\rangle+\right.$

$\left.\left\langle a_{k n-1}, a_{k n-1}^{\prime}\right\rangle\left\langle a_{n-1 n}, a_{n-1 n}^{\prime}\right\rangle\right)\left\langle a_{n-1 n-1}, a_{n-1 n-1}^{\prime}\right\rangle=$

$\left\langle b_{k n}, b_{k n}^{\prime}\right\rangle$, and $\pi(i) \leq \pi(n)$ for each $i \in\{1,2, \ldots, n\}$.

Hence we have shown that $\pi=\left\langle b_{k n}, b_{k n}^{\prime}\right\rangle$.

Similarly, we can prove that $\pi=\left\langle b_{n k}, b_{n k}^{\prime}\right\rangle$ for $k \leq n-2$.

This shows that $\operatorname{adj}(\mathrm{A})$ is idempotent. 
Definition 3.5. (a) We define $P \backslash Q=\{x \in P: x \notin Q\}$. where $P$ and $Q$ be two IFSs

(b) The cardinality of IFS $P$ is denoted by $|P|$

(c) $A=\left(\left\langle a_{i j}, a_{i j}^{\prime}\right\rangle\right) \in M_{n}(F)$ be an idempotent IFM. Let $D(A)=\left\{\left\langle a_{t t}, a_{t t}^{\prime}\right\rangle: t \in\{1,2, \ldots, n\}\right\}$.

We suppose that $|D(A)|=n$ and $\left\langle a_{t t}, a_{t t}^{\prime}\right\rangle \neq\left\langle a_{s s}, a_{s s}^{\prime}\right\rangle$ for $t \neq s$.

We define $\max D(A)=m(1)=\left\langle a_{i_{1}}, a_{i_{1}}^{\prime}\right\rangle$, $\max D(A) \backslash\{m(1)\}=m(2)=\left\langle a_{i_{2}}, a_{i_{2}}^{\prime}\right\rangle$, $D(A) \backslash\{m(1), m(2)\}=m(3)=\left\langle a_{i_{3}}, a_{i_{3}}^{\prime}\right\rangle$ and so on. We have $D(A)=\{m(t): t \in\{1,2, \ldots, n\}\}$. A is said to be an idempotent IFM of T-type if the following condition hold:

(i) $\left\langle a_{i_{1} t}, a_{i_{1} t}^{\prime}\right\rangle \leq m(1)$ and $\left\langle a_{t i_{1}}, a_{t i_{1}}^{\prime}\right\rangle \leq m(1)$ for all $t \in$ $\{1,2, \ldots, n\}$

(ii) $\left\langle a_{i_{2} t}, a_{i_{2} t}^{\prime}\right\rangle \leq m(2)$ and $\left\langle a_{t i_{2}}, a_{t i_{2}}^{\prime}\right\rangle \leq m(2)$ for all $t \in$ $\{1,2, \ldots, n\} \backslash\left\{i_{1}\right\}$

(ii) $\left\langle a_{i_{k} t}, a_{i_{k} t}^{\prime}\right\rangle \leq m(k)$ and $\left\langle a_{t i_{k}}, a_{t i_{k}}^{\prime}\right\rangle \leq m(k)$ for all $t \in$ $\{1,2, \ldots, n\} \backslash\left\{i_{1}, i_{2}, \ldots, i_{k-1}\right\}, k=3,4, \ldots n$

(d) Assume max $D(A)=\left\{\left\langle a_{s s}, a_{s s}^{\prime}\right\rangle,\left\langle a_{t t}, a_{t t}^{\prime}\right\rangle\right\}$.

Then we write $m(1)_{1}=\left\langle a_{s s}, a_{s s}^{\prime}\right\rangle$ and $m(1)_{2}=\left\langle a_{t t}, a_{t t}^{\prime}\right\rangle(s \neq$ $t)$.

Assume max $D(A) \backslash\left\{m(1)_{1}, m(1)_{2}\right\}=$ $\left\{\left\langle a_{i i}, a_{i i}^{\prime}\right\rangle,\left\langle a_{j j}, a_{j j}^{\prime}\right\rangle,\left\langle a_{k k}, a_{k k}^{\prime}\right\rangle\right\}$.

Then we write $m(2)_{1}=\left\langle a_{i i}, a_{i i}^{\prime}\right\rangle, m(2)_{2}=\left\langle a_{j j}, a_{j j}^{\prime}\right\rangle$ and $m(2)_{3}=\left\langle a_{k k}, a_{k k}^{\prime}\right\rangle$.

Similarly, we write $m(3)_{1}=\left\langle a_{u u}, a_{u u}^{\prime}\right\rangle$ and $m(3)_{2}=\left\langle a_{v v}, a_{v v}^{\prime}\right\rangle$, when $\max D(A) \backslash\left\{m(1)_{1}, m(1)_{2}, m(2)_{1}, m(2)_{2}, m(2)_{3}\right\}=$ $\left\{\left\langle a_{u u}, a_{u u}^{\prime}\right\rangle,\left\langle a_{v v}, a_{v v}^{\prime}\right\rangle\right\}$ and so on.

We suppose that $\max d(A) \neq \min D(A)$.

We define idempotent IFM A of T-type as follows: A is said to be T-type if all of the following conditions hold: (i) $\left\langle a_{s x}, a_{s x}^{\prime}\right\rangle \leq m(1)_{1}$ and $\left\langle a_{x s}, a_{x s}^{\prime}\right\rangle \leq m(1)_{1}$ for $x \neq t$, (ii) $\left\langle a_{t x}, a_{t x}^{\prime}\right\rangle \leq m(1)_{2}$ and $\left\langle a_{x t}, a_{x t}^{\prime}\right\rangle \leq m(1)_{2}$ for $x \neq s$, (iii) $\left\langle a_{i x}, a_{i x}^{\prime}\right\rangle \leq m(2)_{1}$ and $\left\langle a_{x i}, a_{x i}^{\prime}\right\rangle \leq m(2)_{1}$ for $x \in$ $\{1,2, \ldots, n\} \backslash\{s, t, j, k\}$,

(iv) $\left\langle a_{j x}, a_{j x}^{\prime}\right\rangle \leq m(2)_{2}$ and $\left\langle a_{x j}, a_{x j}^{\prime}\right\rangle \leq m(2)_{2}$ for $x \in$ $\{1,2, \ldots, n\} \backslash\{s, t, i, k\}$,

$(v)\left\langle a_{k x}, a_{k x}^{\prime}\right\rangle \leq m(2)_{3}$ and $\left\langle a_{x k}, a_{x k}^{\prime}\right\rangle \leq m(2)_{3}$ for $x \in$ $\{1,2, \ldots, n\} \backslash\{s, t, i, j\}$,

(vi) $\left\langle a_{u x}, a_{u x}^{\prime}\right\rangle \leq m(3)_{1}$ and $\left\langle a_{x u}, a_{x u}^{\prime}\right\rangle \leq m(3)_{1}$ for $x \in$ $\{1,2, \ldots, n\} \backslash\{s, t, i, j, k, v\}$,

(vii) $\left\langle a_{v x}, a_{v x}^{\prime}\right\rangle \leq m(3)_{2}$ and $\left\langle a_{x v}, a_{x v}^{\prime}\right\rangle \leq m(3)_{2}$ for $x \in$ $\{1,2, \ldots, n\} \backslash\{s, t, i, j, k, u\}$, and so on.

Lemma 3.6. Let $A \in \mathscr{F}_{n}$ and $n \geq 3$. Then we have that
$\operatorname{adj}\left(E_{i j} A E_{i j}\right)=E_{i j}(\operatorname{adj}(A)) E_{i j}$, where $E_{i j}$ is defined as in Definition 2.4.

Corollary 3.7. Let $A=\left(a_{i j}\right) \in \mathscr{F}_{n}$ be an idempotent IFM of T-type.

We assume that $\max D(A) \neq \min D(A)$. Then $\operatorname{adj}(A)$ is idempotent IFM.

The following example illustrate that the Theorem 3.4 fails if the idempotent IFM is not of T-type.

Example 3.8. $A=\left[\begin{array}{ccc}\langle 0.9,0.1\rangle & \langle 0.0,1.0\rangle & \langle 0.6,0.3\rangle \\ \langle 0.8,0.2\rangle & \langle 0.4,0.5\rangle & \langle 0.6,0.3\rangle \\ \langle 0.1,0.7\rangle & \langle 0.0,1.0\rangle & \langle 0.2,0.6\rangle\end{array}\right]$

$A^{2}=\left[\begin{array}{ccc}\langle 0.9,0.1\rangle & \langle 0.0,1.0\rangle & \langle 0.6,0.3\rangle \\ \langle 0.8,0.2\rangle & \langle 0.4,0.5\rangle & \langle 0.6,0.3\rangle \\ \langle 0.1,0.7\rangle & \langle 0.0,1.0\rangle & \langle 0.2,0.6\rangle\end{array}\right]$

Therefore $A$ is an idempotent IFM but not of T-type

Let $\operatorname{adj}(A)=B$ we get

$\operatorname{adj}(A)=B=\left[\begin{array}{ccc}\langle 0.2,0.6\rangle & \langle 0.0,1.0\rangle & \langle 0.4,0.5\rangle \\ \langle 0.2,0.6\rangle & \langle 0.2,0.6\rangle & \langle 0.6,0.3\rangle \\ \langle 0.1,0.7\rangle & \langle 0.0,1.0\rangle & \langle 0.4,0.5\rangle\end{array}\right]$

$B^{2}=\left[\begin{array}{ccc}\langle 0.2,0.6\rangle & \langle 0.0,1.0\rangle & \langle 0.4,0.5\rangle \\ \langle 0.2,0.6\rangle & \langle 0.2,0.6\rangle & \langle 0.4,0.5\rangle \\ \langle 0.1,0.7\rangle & \langle 0.0,1.0\rangle & \langle 0.4,0.5\rangle\end{array}\right]$

$\Rightarrow B^{2} \neq B$

Therefore $B$ is not an idempotent IFM.

The following example shows that if $A$ is an idempotent IFM of the T-type and $A$ is normal then the Theorem 3.4 holds.

Example 3.9. $A=\left[\begin{array}{ccc}\langle 0.8,0.2\rangle & \langle 0.6,0.3\rangle & \langle 0.4,0.5\rangle \\ \langle 0.2,0.7\rangle & \langle 0.4,0.5\rangle & \langle 0.3,0.6\rangle \\ \langle 0.1,0.8\rangle & \langle 0.1,0.8\rangle & \langle 0.1,0.8\rangle\end{array}\right]$

$A^{2}=\left[\begin{array}{ccc}\langle 0.8,0.2\rangle & \langle 0.6,0.3\rangle & \langle 0.4,0.5\rangle \\ \langle 0.2,0.7\rangle & \langle 0.4,0.5\rangle & \langle 0.3,0.6\rangle \\ \langle 0.1,0.8\rangle & \langle 0.1,0.8\rangle & \langle 0.1,0.8\rangle\end{array}\right]$

$\Rightarrow A^{2}=A$

Therefore $A$ is an idempotent IFM of T-type and also A is normal.

Now,

$\operatorname{adj}(A)=B$

$\operatorname{adj}(A)=B=\left[\begin{array}{ccc}\langle 0.1,0.8\rangle & \langle 0.1,0.8\rangle & \langle 0.4,0.5\rangle \\ \langle 0.1,0.8\rangle & \langle 0.1,0.8\rangle & \langle 0.3,0.6\rangle \\ \langle 0.1,0.8\rangle & \langle 0.1,0.8\rangle & \langle 0.4,0.5\rangle\end{array}\right]$

$B^{2}=\left[\begin{array}{ccc}\langle 0.1,0.8\rangle & \langle 0.1,0.8\rangle & \langle 0.4,0.5\rangle \\ \langle 0.1,0.8\rangle & \langle 0.1,0.8\rangle & \langle 0.3,0.6\rangle \\ \langle 0.1,0.8\rangle & \langle 0.1,0.8\rangle & \langle 0.4,0.5\rangle\end{array}\right]$

$\Rightarrow B^{2}=B$

Therefore $B$ is an idempotent IFM. 


\section{Conclusion}

In this paper we have defined normal IFM and idempotent IFMs of T-type. Further, we have developed a result on idempotent IFM and IFMs of T-type.

\section{Conflict of Interest}

No potential conflict of interest relevant to this article was reported.

\section{References}

[1] L. A. Zadeh, "Fuzzy sets," Information and Control, vol. 8, no. 3, pp. 338-353, 1965. http://dx.doi.org/10.1016/S00199958(65)90241-X

[2] K. T. Atanassov, "Intuitionistic fuzzy sets," Fuzzy Sets and Systems, vol. 20, no. 1, pp. 87-96, 1986. http://dx.doi. org/10.1016/S0165-0114(86)80034-3

[3] L. C. Jang, W. J. Kim, and T. Kim, "A note on distances between interval-valued intuitionistic fuzzy sets," International Journal of Fuzzy Logic and Intelligent Systems, vol. 11, no. 1, pp. 8-11, 2011. http://dx.doi.org/10.5391/IJFIS. 2011.11.1.008

[4] J. H. Park, Y. C. Kwun, and M. J. Son, "A generalized intuitionistic fuzzy soft set theoretic approach to decision making problems," International Journal of Fuzzy Logic and Intelligent Systems, vol. 11, no. 2, pp. 71-76, 2011. http://dx.doi.org/10.5391/IJFIS.2011.11.2.071

[5] K. H. Kim and F. W. Roush, "Generalized fuzzy matrices," Fuzzy Sets and Systems, vol. 4, no. 3, pp. 293-315, 1980. http://dx.doi.org/10.1016/0165-0114(80)90016-0

[6] M. G. Thomason, "Convergence of powers of a fuzzy matrix," Journal of Mathematical Analysis and Applications, vol. 57, no. 2, pp. 476-480, 1977. http://dx.doi.org/ 10.1016/0022-247X(77)90274-8

[7] J. B. Kim, "Idempotents and inverse in fuzzy matrices," Bulletin of the Malaysian Mathematical Sciences Society, vol. 6, no. 2, pp. 57-61, 1983.

[8] M. A. Mishref and E. G. Emam, "Transitivity and subinverses in fuzzy matrices," Fuzzy Sets and Systems, vol. 52, no. 3, pp. 337-343, 1992. http://dx.doi.org/10.1016/01650114(92)90241-U
[9] M. Z. Ragab and E. G. Emam, "The determinant and adjoint of a square fuzzy matrix," Information Sciences, vol. 84, no. 3-4, pp. 209-220, 1995. http://dx.doi.org/10. 1016/0020-0255(93)00064-5

[10] J. B. Kim, "On fuzzy idempotent matrices of T-Type," Information Sciences, vol. 80, no. 3-4, pp. 311-318, 1994. http://dx.doi.org/10.1016/0020-0255(94)90082-5

[11] A. R. Meenakshi, Fuzzy Matrix: Theory and Applications. Chennai: MJP Publishers, 2008.

[12] M. Pal, "Intuitionistic fuzzy determinant," V.U.J. Physical Sciences, vol. 7, pp. 87-93, 2001.

[13] Y. B. Im, E. P. Lee, and S. W. Park, "The determinant of square intuitionistic fuzzy matrices," Far East Journal of Mathematical Sciences, vol. 3, no. 5, pp. 789-796, 2001.

[14] M. Pal, S. K. Khan, and A. K. Shyamal, "Intuitionistic fuzzy matrices," Notes on Intuitionistic Fuzzy Sets, vol. 8, no. 2, pp. 51-62, 2002.

[15] A. K. Shyamal and M. Pal, "Two new operators on fuzzy matrices," Journal of Applied Mathematics and Computing, vol. 15, no. 1, pp. 91-107, 2004. http://dx.doi.org/10. 1007/BF02935748

[16] A. K. Shyamal and M. Pal, "Distances between intuitionistics fuzzy matrices," V.U.J. Physical Sciences, vol. 8, pp. 81-91, 2002.

[17] M. Bhowmik and M. Pal, "Generalized intuitionistic fuzzy matrices," Far East Journal of Mathematical Science, vol. 29, no. 3, pp. 533-554, 2008

[18] M. Bhowmik and M. Pal, "Generalized interval-valued intuitionistic fuzzy sets," The Journal of Fuzzy Mathematics, vol. 18 , no. 2 , pp. $357-371,2010$.

[19] M. Bhowmik and M. Pal, "Some results on generalized interval-valued intuitionistic fuzzy sets," International Journal of Fuzzy Systems, vol. 14, no. 2, pp. 193-203, 2012.

[20] S. K. Khan and A. Pal, "The generalized inverse of intuitionistic fuzzy matrices," Journal of Physical Sciences, vol. 11, pp. 62-67, 2007.

[21] A. K. Adak, M. Bhowmik, and M. Pal, "Some properties of generalized intuitionistic fuzzy nilpotent matrices over distributive lattice," Fuzzy Information and Engineering, 
vol. 4, no. 4, pp. 371-387, 2012. http://dx.doi.org/10.1007/ s12543-012-0121-1

[22] R. A. Padder and P. Murgadas, "Max-max operation on intuitionistic fuzzy matrix," Annals of Fuzzy Mathematics and Informatics, in press.

[23] P. Murugadas and R. A. Padder, "Reduction of an intuitionistic fuzzy rectangular Matrix," Annamalai University Science Journal, vol. 49, pp. 15-18, 2015.

[24] R. Pradhan and M. Pal, "Convergence of maxarithmetic mean-minarithmetic mean powers of intuitionistic fuzzy matrices," International Journal of Fuzzy Mathematical Archive, vol. 2, pp. 58-69, 2013.

[25] S. Sriram and P. Murugadas, "On semiring of intuitionstic fuzzy matrices," Applied Mathematical Sciences, vol. 4, no. 23, pp. 1099-1105, 2010.

[26] S. Sriram and P. Murugadas, "Sub-inverses of intuitionistic fuzzy matrices," Acta Ciencia Indica Mathematics, vol. 37, no. 1, pp. 41-56, 2011.

[27] H. Y. Lee and N. G. Jeong, "Canonical form of a transitive intuitionistic fuzzy matrices," Honam Mathematical Journal, vol. 27 no. 4, pp. 543-550, 2005.

[28] K. Atanassov, "Generalized index matrices," Comptes Rendus de L'academie Bulgare des Sciences, vol. 40, no. 11, pp. 15-18, 1987.
[29] K. T. Atanassov, "Intuitionistic fuzzy sets," in Proceedings of the Polish Symposium on Interval and Fuzzy Mathematics (VII ITKR's Session), Sofia, 1983.

[30] Y. B. Im, E. P. Lee, and S. W. Park, "The adjoint of square intuitionistic fuzzy matrices," Journal of Applied Mathematics and Informatics, vol. 11, no. 1-2, pp. 401-412, 2003.

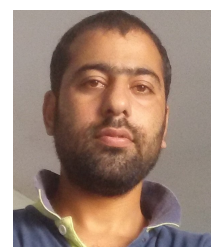

Riyaz Ahmad Padder received M.Sc. Mathematics from Barkatullah vishwavidylaya University in 2011. Since 2013, he is pursuing Ph.D. degree in the department of Mathematics Annamalai University. His area of interest include fuzzy matrix theory and intuitionistic fuzzy matrix theory.

E-mail: padderriyaz01@gmail.com

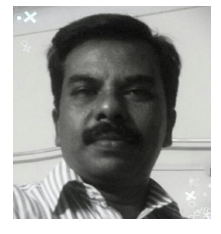

P. Murugadas received his M.S., M.Phil in Mathematics from Maduraj Kamaraj University, Ph.D., in Mathematics from Annamalai university Tamil Nadu, in 1987, 1988 and 2011, respectively. He joined Annamalai University, India in 2006 and is currently as Assistant Professor in the Department of Mathematics. His research interests include fuzzy algebra, fuzzy set theory, fuzzy matrix theory, intuitionistic fuzzy set theory and intuitionistic fuzzy matrix.

E-mail: bodi_muruga@yahoo.com 